\title{
Metformin: clinical topics and new mechanisms of action
}

\author{
Yoshihito Fujita $^{1}$ - Nobuya Inagaki ${ }^{1}$
}

Received: 24 November 2016/Published online: 9 December 2016

(C) The Japan Diabetes Society 2016

Keywords Metformin - Preparation - Chronic kidney disease $\cdot$ Nonglycemic effect $\cdot$ Anticancer effect

Metformin is a widely used orally administerd glucoselowering drug for type 2 diabetes and is recommended as a first-line drug in recent treatment guidelines of the American Diabetes Association (ADA) and European Association for the Study of Diabetes (EASD) [1]. Metformin is derived from the plant Galega officinalis (French lilac) traditionally used in Europe for diabetes treatment [2]. The main target tissue of metformin is the liver, and its major effect is to decrease hepatic glucose output largely by suppressing gluconeogenesis, which leads to lower fasting blood glucose levels without insulin stimulation and weight gain [3]. Despite metformin being first introduced as a treatment for type 2 diabetes in 1957, it's mechanism of action is not yet fully understood.

In recent decades, new mechanisms of metformin action have been proposed, along with improvements in molecular research techniques. Metformin has an inhibitory effect on mitochondrial complex I, inhibition of which increases the adenosine monophosphate/adenosine triphosphate (AMP/ATP) ratio $[4,5]$. The altered cellular energy status induces activation of AMP-activated protein kinase (AMPK), a serine/threonine kinase, and acts as an energy sensor [6]. Zhou et al. demonstrated that the suppressing effect of metformin on hepatic gluconeogenesis is mediated by AMPK activation [7]. Various mechanisms by

Nobuya Inagaki

inagaki@kuhp.kyoto-u.ac.jp

1 Department of Diabetes, Endocrinology and Nutrition, Graduate School of Medicine, Kyoto University, 54 Shogoin, Kawahara-cho, Sakyo-ku, Kyoto 606-8507, Japan which metformin activates AMPK are proposed, some of which are mediation due to increased AMP by inhibition of AMP deaminase, and mediation by activation of endothelial nitric oxide synthase (eNOS) [8, 9]. Shaw et al. reported that liver kinase B1 (LKB1), an upstream kinase of AMPK, participates in metformin action by activating AMPK and regulating gluconeogenic enzymes [10]. AMPK-independent mechanisms are also proposed. Miller et al. reported a suppressing effect of hepatic glucagon signaling via inhibition of adenylyl cyclase activity that participates in metformin action [11]. Madiraju et al. reported that metformin inhibits mitochondrial glycerophosphate dehydrogenase (mGPD), a glycerophosphate shuttle enzyme, to exert a suppressing effect on hepatic gluconeogenesis [12].

Although the liver is considered the main target tissue of metformin, recent basic and clinical studies indicate the gut as an important site of its action, as short-term metformin administration IV is less effective than oral administration in rats and humans [13]. Metformin performs a number of actions within the gut [14]. It increases glucose uptake, anaerobic glucose utilization, lactate production in the intestine; secretion of the enteroendocrine L-cell products glucagon-like peptide 1 (GLP-1) and peptide YY (PYY), and influences the gut-brain axis, bile acid metabolism, and gut microbiome. Each of these mechanisms has been proposed as a contributing factor in its direct and indirect glucose-lowering action.

Recently, new preparations of metformin have been developed for possible improvements in efficiency and tolerability, expanding its clinical indications. The conventional preparation, immediate release (IR), has been used for more than 5 decades and requires dosing two or three times daily, which inhibits drug compliance and results in a high frequency of gastrointestinal (GI) side 
effects, which inhibit tolerability [15]. The extended-release formulation (Metformin XR) is in use clinically and enables slower drug absorption in the upper GI tract using a once-daily dosing option, and the frequency and severity of GI side effects is lower [16]. Delayed-release metformin (Metformin DR) also provides a once-daily dosing option and was developed to maximize gut-based mechanisms of action by targeting the drug to the ileum [17, 18]. Metformin DR comprises a metformin IR hydrochloride $(\mathrm{HCl})$ core overlaid with a proprietary enteric coat, which delays disintegration and dissolution of the tablet until it reaches $\mathrm{pH}$ of 6.5 in the distal small intestine and beyond, thus bypassing the usual, major sites of absorption. Clinical studies using metformin DR highlights the ileum as a site of uptake and as an important site of action in lowering blood glucose. Compared with metformin IR or metformin $\mathrm{XR}$, the bioavailability of metformin DR is lower, yet its glucose-lowering efficacy is similar despite the lower systemic metformin exposure [19]. After a single daily doseof DR $1000 \mathrm{mg}$, plasma concentrations and bioavailability were $\sim 50 \%$ compared with those using XR; however, clinical effects after 4 weeks were similar. In addition, while the extent of systemic metformin exposure is reduced by $45 \%$ with twice-daily DR $1000 \mathrm{mg}$ compared with twice-daily IR $1000 \mathrm{mg}$, both regimens result in a similar increase in gut hormones, such as GLP-1 and PYY [20]. A potential advance provided by metformin DR may be the use of biguanide for patients with chronic kidney disease (CKD) and those at higher risk of lactic acidosis [18]. Phase III efficacy and safety studies of DR vs. placebo or IR are now planned in patients with renal impairment.

The U.S. Food and Drug Administration (FDA) approved metformin for clinical use in 1995. At that time labeling included a contraindication for patients with moderate to severe renal impairment. Subsequently, studies found it could be safely used in patients with mild to moderate renal impairment [21, 22], and in 2016, the FDA revised its warnings accordingly to include patients with an estimated glomerular filtration rate (eGFR) between 30 and $60 \mathrm{ml} / \mathrm{min} / 1.73 \mathrm{~m}^{2}$ [23].

Metformin is also known to have numerous nonglycemic effects. In the UK Prospective Diabetes Study (UKPDS), metformin had a robust effect on cardiovascular risk [24, 25]. Improved cardiovascular outcome was not observed in patients randomized to receive intensive glycemic management with sulfonylurea or insulin, however, suggesting the potential of metformin to improve cardiovascular outcome independent of glycemic control. Based largely on the findings of UKPDS, metformin has emerged as the first-line therapy for treating type 2 diabetes (ADA and EASD) [1]. Clinical data and data from animal studies support a direct protective action on the vascular endothelium from metformin, and different mechanisms beyond glycemic control have been implicated: improvements in the inflammatory pathway, coagulation, oxidative stress, endothelial dysfunction, and hemostasis [3].

Patients with diabetes have a higher risk of liver, pancreas, breast, and colon cancers [26], with an incidence estimated to be about 1.2 times higher than in nondiabetic individuals [27]. Observational epidemiologic studies suggest that some antidiabetic medications might affect cancer risk; the anticancer effect of metformin has recently received much attention [3]. On the other hand, epidemiologic studies present conflicting conclusions, and a metaanalysis of available randomized controlled trial data does not support the hypothesis that metformin lowers cancer risk. Eligible trials also showed no significant effect of metformin on all-cause mortality [28]. Nevertheless, metformin has been tried with some success in clinical chemotherapy trials for treatment of various types of cancer, and many trials are ongoing, including for breast, prostate, colorectal, pancreas, and lung cancers [29].

Other nonglycemic effects of metformin have also been reported, most being associated with ameliorating effects on insulin resistance, such as polycystic ovary syndrome (PCOS) and nonalcoholic steatohepatitis (NASH)/nonalcoholic fatty liver disease (NAFLD) [15]. Further investigations and randomized controlled trials in nondiabetic individuals are required to demonstrate the nonglycemic effects of metformin.

Acknowledgements This work was supported by Scientific Research Grants from the Ministry of Education, Culture, Sports, Science, and Technology of Japan. Nobuya Inagaki received research grants from Astellas Pharma Inc., Taisho Toyama Pharmaceutical Co., Ltd., Mitsubishi Tanabe Pharma Corporation, Takeda Pharmaceutical Company Limited, Daiichi Sankyo Company, Limited, MSD, Sanofi, Dainippon Sumitomo Pharma Co., Ltd., Kyowa Hakko Kirin Co., Ltd., Eli Lilly Japan K.K., Shiratori Pharmaceutical Co., Ltd., Ono Pharmaceutical Co., Ltd., JT, Pfizer, Nippon Boehringer Ingelheim Co., Ltd., Sanwa Kagaku Kenkyusho Co., Ltd., Kissei Pharmaceutical Co., Ltd., AstraZeneca, and Japan Diabetes Foundation.

\section{Compliance with ethical standards}

Conflict of interest Yoshihito Fujita declares no conflict of interest.

Ethics policy This article does not report any studies with human or animal subjects that were performed by any of the authors.

\section{References}

1. Inzucchi SE, Bergenstal RM, Buse JB, et al. Management of hyperglycemia in type 2 diabetes, 2015: a patient-centered approach: update to a position statement of the American Diabetes Association and the European Association for the Study of Diabetes. Diabetes Care. 2015;38:140-9.

2. Rojas LB, Gomes MB. Metformin: an old but still the best treatment for type 2 diabetes. Diabetol Metab Syndr. 2013; 5:6. 
3. Pryor R, Cabreiro F. Repurposing metformin: an old drug with new tricks in its binding pockets. Biochem J. 2015;471:307-22.

4. El-Mir MY, Nogueira V, Fontaine E, et al. Dimethylbiguanide inhibits cell respiration via an indirect effect targeted on the respiratory chain complex I. J Biol Chem. 2000; 275:223-28.

5. Owen MR, Doran E, Halestrap AP. Evidence that metformin exerts its antidiabetic effects through inhibition of complex 1 of the mitochondrial respiratory chain. Biochem J. 2000;348:607-14.

6. Hardie DG. The AMP-activated protein kinase pathway-new players upstream and downstream. J Cell Sci. 2004;117:5479-87.

7. Zhou G, Myers R, Li Y, Chen Y, et al. Role of AMP-activated protein kinase in mechanism of metformin action. J Clin Invest. 2001; 108:1167-74.

8. Ouyang JY, Parakhia RA, Ochs RS. Metformin activates AMP kinase through inhibition of AMP deaminase. J Biol Chem. 2011;286:1-11.

9. Fujita Y, Hosokawa M, Fujimoto S, et al. Metformin suppresses hepatic gluconeogenesis and lowers fasting blood glucose levels through reactive nitrogen species in mice. Diabetologia. 2010;53:1472-81.

10. Shaw RJ, Lamia KA, Vasquez D, et al. The kinase LKB1 mediates glucose homeostasis in liver and therapeutic effects of metformin. Science. 2005; 310:1642-46.

11. Miller RA, Chu Q, Xie J, Foretz M, et al. Biguanides suppress hepatic glucagon signalling by decreasing production of cyclic AMP. Nature. 2013;494:256-60.

12. Madiraju AK, Erion DM, Rahimi Y, et al. Metformin suppresses gluconeogenesis by inhibiting mitochondrial glycerophosphatedehydrogenase. Nature. 2014;510:542-6.

13. Bonora E, Cigolini M, Bosello O, et al. Lack of effect of intravenous metformin on plasma concentrations of glucose, insulin, C-peptide, glucagon and growth hormone in nondiabetic subjects. Curr Med Res Opin. 1984;9:47-51.

14. McCreight LJ, Bailey CJ, Pearson ER. Metformin and the gastrointestinal tract. Diabetologia. 2016;59:426-35.

15. Campbell IW, Bailey C, Bailey CJ, et al. Metformin-the gold standard: a scientific handbook. 2008. New York: Wiley.

16. Levy J, Cobas RA, Gomes MB. Assessment of efficacy and tolerability of oncedaily extended release metformin in patients with type 2 diabetes mellitus. Diabetol Metab Syndr. 2010;2:16.

17. Scheen AJ. Will delayed release metformin provide better management of diabetes type 2? Expert Opin Pharmacother. 2016;17:627-30.
18. DeFronzo R, Fleming GA, Chen K, et al. Metformin-associated lactic acidosis: current perspectives on causes and risk. Metabolism. 2016;65:20-9.

19. Buse JB, DeFronzo RA, Rosenstock J, et al. The primary glucoselowering effect of metformin resides in the gut, not the circulation: results from short-term pharmacokinetic and 12-week doseranging studies. Diabetes Care. 2016;39:198-205.

20. DeFronzo RA, Buse JB, Kim T, et al. Once-daily delayed-release metformin lowers plasma glucose and enhances fasting and postprandial GLP-1 and PYY: results from two randomised trials. Diabetologia. 2016;59:1645-54.

21. Roussel R, Travert F, Pasquet B, et al. Metformin use and mortality among patients with diabetes and atherothrombosis. Arch Intern Med. 2010;170:1892-9.

22. Ekström N, Schiöler L, Svensson AM, et al. Effectiveness and safety of metformin in 51675 patients with type 2 diabetes and different levels of renal function: a cohort study from the Swedish National Diabetes Register. BMJ Open. 2012; 2.

23. FDA Drug Safety Communication. FDA revises warnings regarding use of the diabetes medicine metformin in certain patients with reduced kidney function. http://www.fda.gov/ Drugs/DrugSafety/ucm493244.htm. Accessed 4 Aug 2016.

24. UKPDS Group. Effect of intensive blood-glucose control with metformin on complications in overweight patients with type 2 diabetes (UKPDS 34). Lancet. 1998; 352:854-65.

25. Holman R, Paul S, Bethel M, Matthews D, Neil H. 10-year follow-up of intensive glucose control in type 2 diabetes. $\mathrm{N}$ Engl $\mathrm{J}$ Med. 2008;359:1577-89.

26. Giovannucci E, Harlan DM, Archer MC, et al. Diabetes and cancer: a consensus report. Diabetes Care. 2010;33:1674-85.

27. Noto H, Tsujimoto T, Noda M. Significantly increased risk of cancer in diabetes mellitus patients: a meta-analysis of epidemiological evidence in Asians and nonAsians. J Diabetes Investig. 2012;3:24-33.

28. Stevens RJ, Ali R, Bankhead CR, et al. Cancer outcomes and allcause mortality in adults allocated to metformin: systematic review and collaborative meta-analysis of randomised clinical trials. Diabetologia. 2012; 55:2593-603.

29. Chae YK, Arya A, Malecek MK, et al. Repurposing metformin for cancer treatment: current clinical studies. Oncotarget. 2016;26:40767-80. 